\title{
Pickover Biomorphs and Non-standard Complex Numbers
}

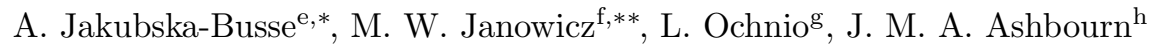 \\ ${ }^{a}$ Department of Botany, Institute of Environmental Biology, University of Wrocaw, \\ Kanonia 6/8, 50-328 Wroclaw, Poland \\ ${ }^{b}$ Department of Applied Mathematics, Faculty of Applied Computer Science and \\ Mathematics, Warsaw University of Life Sciences - SGGW, ul. Ciszewskiego 8, 02-786 \\ Warsaw, Poland \\ ${ }^{c}$ Department of Econometrics and Statistics, Faculty of Applied Computer Science and \\ Mathematics, Warsaw University of Life Sciences - SGGW, ul. Nowoursynowska 159, \\ 02-787 Warsaw, Poland \\ ${ }^{d}$ St Cross College, University of Oxford, St Giles', Oxford OX1 3LZ, United Kingdom
}

Keywords: Pickover biomorphs, self-similar structures, fractals, hyperbolic complex numbers, Moran autocorrelation function, sample entropy, Hurst exponent

\footnotetext{
${ }^{*}$ Corresponding author

${ }^{* *}$ Corresponding author

Email addresses: anna.jakubska-busse@uwr.edu.pl (A. Jakubska-Busse), maciej_janowicz@sggw.pl (M. W. Janowicz)
} 


\title{
Pickover Biomorphs and Non-standard Complex Numbers
}

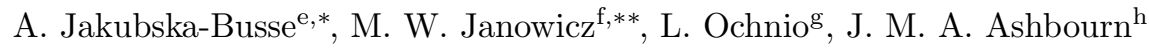 \\ ${ }^{e}$ Department of Botany, Institute of Environmental Biology, University of Wrocaw, \\ Kanonia 6/8, 50-328 Wroclaw, Poland \\ ${ }^{f}$ Department of Applied Mathematics, Faculty of Applied Computer Science and \\ Mathematics, Warsaw University of Life Sciences - SGGW, ul. Ciszewskiego 8, 02-786 \\ Warsaw, Poland \\ ${ }^{g}$ Department of Econometrics and Statistics, Faculty of Applied Computer Science and \\ Mathematics, Warsaw University of Life Sciences - SGGW, ul. Nowoursynowska 159, \\ 02-787 Warsaw, Poland \\ ${ }^{h}$ St Cross College, University of Oxford, St Giles', Oxford OX1 3LZ, United Kingdom
}

\begin{abstract}
In this study Pickover biomorphs are analysed as being dependent on the chosen complex number system in which iterations of analytic functions are performed. Moran's spatial autocorrelation function and two forms of entropy, Shannon's and the sample entropy, are chosen in order to find correlations and measure complexity in Pickover biomorphs. These turn out to be strongly correlated and low-entropy objects with a fractal dimension between 1.4 and 2 . It is shown that there is a strong maximum in correlation and a strong minimum in entropy for the case of Galilean complex numbers corresponding to the square of the generalised imaginary unit being equal to zero.
\end{abstract}

\section{Introduction}

Mandelbrot sets, Julia sets $[1,2,3,4]$ and Pickover biomorphs [5, 6, 7, $8,9]$ belong to a group of mathematical and graphical fractal objects which are of particular fascination to both scientists and artists. Remarkably some Pickover biomorphs morphologically resemble unicellular protozoa with their various organelles or even aquatic crustaceans. Together with better known related objects such as Mandelbrot sets, Pickover biomorphs are an interesting example of very complex structures emerging from the application of simple rules which determine the application of a transformation of the complex plane by iterations of analytic functions. Due to the simplicity of such rules, Pickover biomorphs have attracted some mild attention in the field of theoretical biology

\footnotetext{
*Corresponding author

${ }^{* *}$ Corresponding author

Email addresses: anna.jakubska-busse@uwr.edu.pl (A. Jakubska-Busse), maciej_janowicz@sggw.pl (M. W. Janowicz)
} 
$[10,11]$. These applications in the simulation of evolution set out the most important context of the present study. In one sense, Pickover biomorphs may also be said to be a fascinating by-product of complex dynamics in mathematics $[12]$.

It is not, however, easy to analyse Pickover biomorphs mathematically partly because (to the authors' best knowledge) there is not even a heuristic mathematical definition. Pickover biomorphs are in fact defined by a family of algorithms which create them or even directly as merely graphical objects which resemble certain invertebrates. Thus, this study solely relates to images obtained from more or less standard algorithms. In order to characterise biomorphs quantitatively, two kinds of measures have been used. Firstly, as a measure of spatial correlations, the Moran autocorrelation function has been chosen. Secondly, as a measure of complexity, two versions of entropy, Shannon's and the sample entropy have been applied. The second type of entropy is considered to be an approximation of the Kolmogorov-Sinai entropy. It turns out that even though the autocorrelation function and the entropy measure different characteristics of the biomorphs they still seem to be complementary to each other.

There are two obvious considerations at this point, namely, (a) whether it is of any use to extend the notion of biomorphs to non-standard systems of complex numbers, and (b) what exactly the purpose of application of the machinery of statistics and complexity-related measures to them is. For (a), we believe that from the point of view of investigations in evolution theory exemplified by [10] it is useful to have at one's disposal structures with sharply defined qualitative changes of properties which can be obtained just by changing one very well defined parameter, in our case here the square of the imaginary unit. For (b), when simulating the evolution of simple forms with the help of genetic algorithms as in [10] it is important to know whether the "evolution" leads to greater or smaller complexity, stronger or weaker morphological correlations, and even lower or higher fractal dimensions.

\section{Non-standard Systems of Complex Numbers}

It is well known that one of the fundamentally important procedures in mathematics is the pairing of two real numbers to form a complex number. Multiplication in the set of complex numbers can be defined using Hamilton's construction or alternatively, any complex number $z$ can be written as $z=x+i y$ with $i^{2}=-1$. One can then formally operate with complex numbers as with real ones under the assumption of distributiveness of multiplication with respect to addition and substituting in for $i^{2}$. Let us note that if $i$ is understood as just a symbol (rather than a number) with a defined square, the construction of complex numbers can easily be extended to cases where $i^{2}$ is different to -1 . In fact there exist three canonical complex number systems corresponding to $i^{2}=-1,0$ and +1 . Following [13], these will be referred to here as standard (Euclidean), Galilean, and hyperbolic (complex) numbers respectively. In the case of Galilean complex numbers [14] the law of multiplication of two numbers 
is:

$$
(a+b i)(c+d i)=a c+(a d+b c) i,
$$

and in the case of hyperbolic complex numbers the law takes the form:

$$
(a+b i)(c+d i)=(a c+b d)+(a d+b c) i .
$$

An important point here is that the concept of analytic (or holomorphic) function as a function which depends only on $z=a+b i$ but not on $z^{\star}=a-b i$ can be naturally extended to the case of Galilean and hyperbolic numbers. The Cauchy-Riemann equations as necessary conditions of analyticity can also be easily formulated.

It can be noted that hyperbolic complex numbers seem to be particularly interesting from the point of view of theoretical physics. Indeed, a reasonably far-reaching theory of analytic functions can be built based on their set. The corresponding Cauchy-Riemann equations are no more than the free Maxwell equations in $(1+1)$ dimensions. Taking into account that every analytic function provides solutions to the Cauchy-Riemann equations, solutions to $(1+1)$ -dimensional Maxwell equations can be obtained, which might satisfy interesting boundary conditions [13]. This could be of interest, for example, from the point of view of cavity quantum electrodynamics with moving boundaries where exact oscillating solutions to the Maxwell equations are important (see, e.g. [15], [16], $[17])$.

\section{Results}

In the first part of this section, several pictures of biomorphs obtained from the iterations of almost the simplest possible analytic functions, namely quadratic and cubic ones, are presented. The second part is devoted to their analysis using well-defined statistical and complexity measures. In the latter part, the study has been deliberately restricted to such simple functions in order to avoid dealing with too much scattered data with the loss of necessary focus.

\subsection{Standard Pickover Biomorphs from Iteration of a Cubic Function}

Let us consider the following iteration:

$$
z \longleftarrow z^{n}+C
$$

where $\longleftarrow$ is the assignment operator (as in [18]) and we then introduce the following criterion to stop the iteration procedure:

$$
\operatorname{Re}(z)^{2}>r_{0} \text { or } \operatorname{Im}(z)^{2}>r_{0} .
$$

It is precisely because of the seemingly innocent "or" statement in the above criterion that the images produced by the appropriate colouring of the structure 
obtained differ radically from otherwise closely related Mandelbrot or Julia sets. Examples of standard Pickover biomorphs obtained from the above procedure with $n=2$ and $n=3$ are shown in Figure 1. The "simulation cell" has a dimension of $1024 x 1024$ pixels and the starting points have been located in the square $-2.5 \leq \operatorname{Re}(z) \leq 2.5,-2.5 \leq \operatorname{Im}(z) \leq 2.5$. In all examples shown below the maximal number of iterations has been equal to 100 .

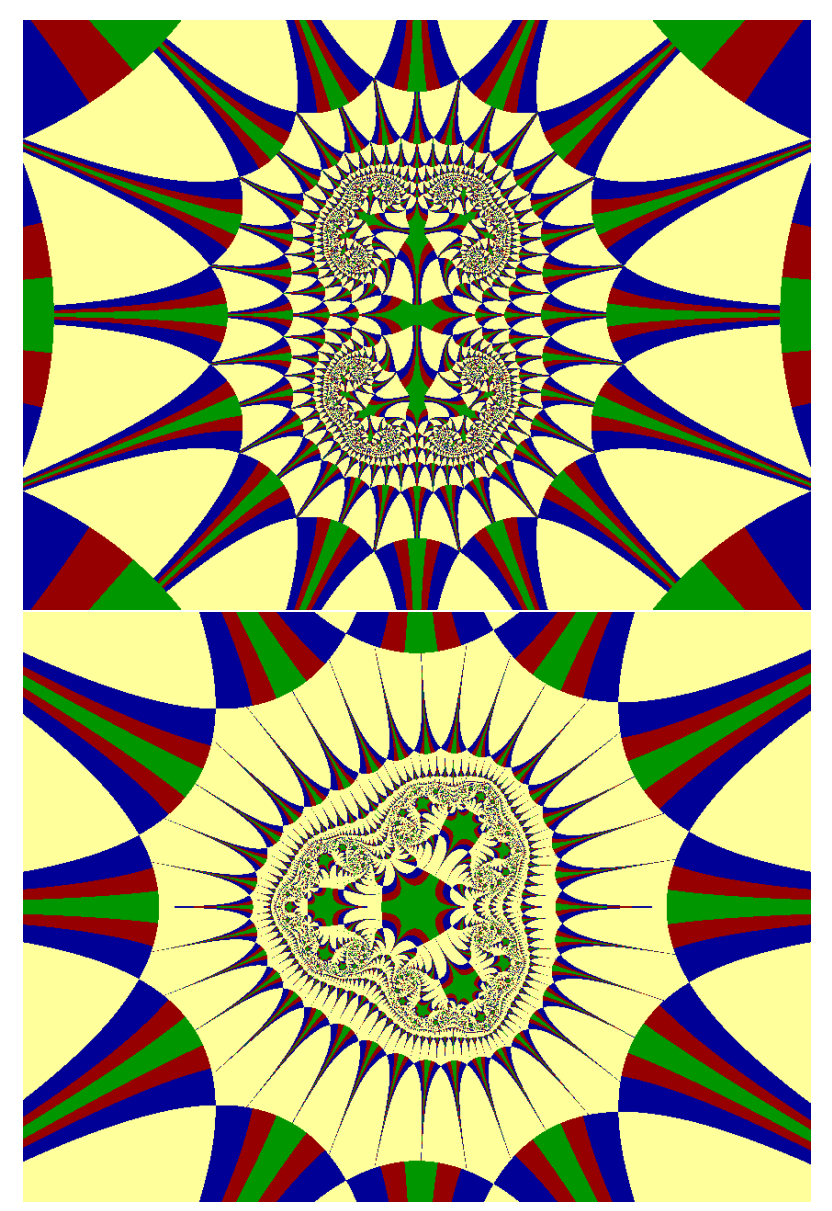

Figure 1: Standard Pickover biomorphs obtained from the iteration of quadratic (upper) and cubic (lower) functions.

The value of $r_{0}$ has been set equal to 10.0 and $C$ has been set equal to 0.5 (i.e. with a zero imaginary part).

\subsection{Pickover Biomorphs in the Realm of Galilean Numbers}

The same quadratic and cubic iteration schemes and parameters are applied to Galilean complex numbers and result in the image shown in Figure 2: 


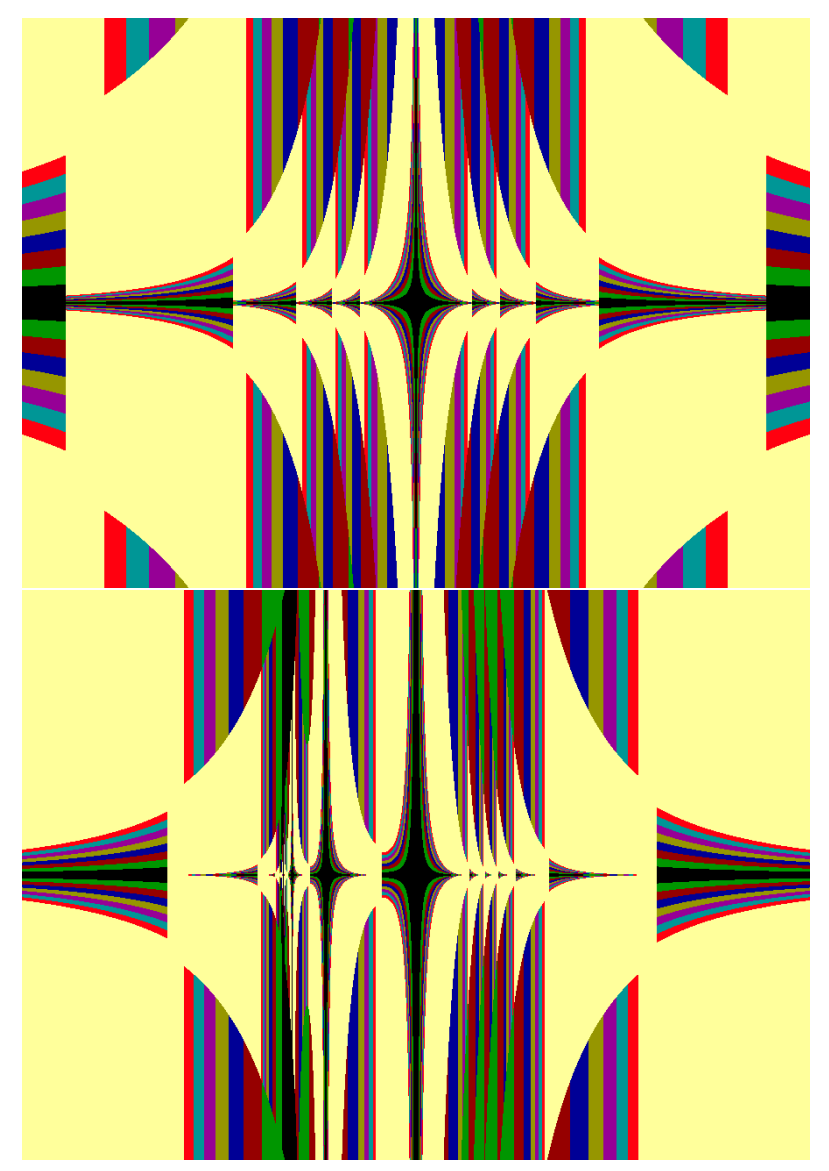

Figure 2: Pickover biomorphs for Galilean complex numbers obtained from the iteration of quadratic (upper) and cubic (lower) functions.

\subsection{Pickover Biomorphs and Hyperbolic Complex Numbers}

If it is now assumed that $i^{2}=+1$, the Pickover biomorphs take very different forms as illustrated in Figure 3. 


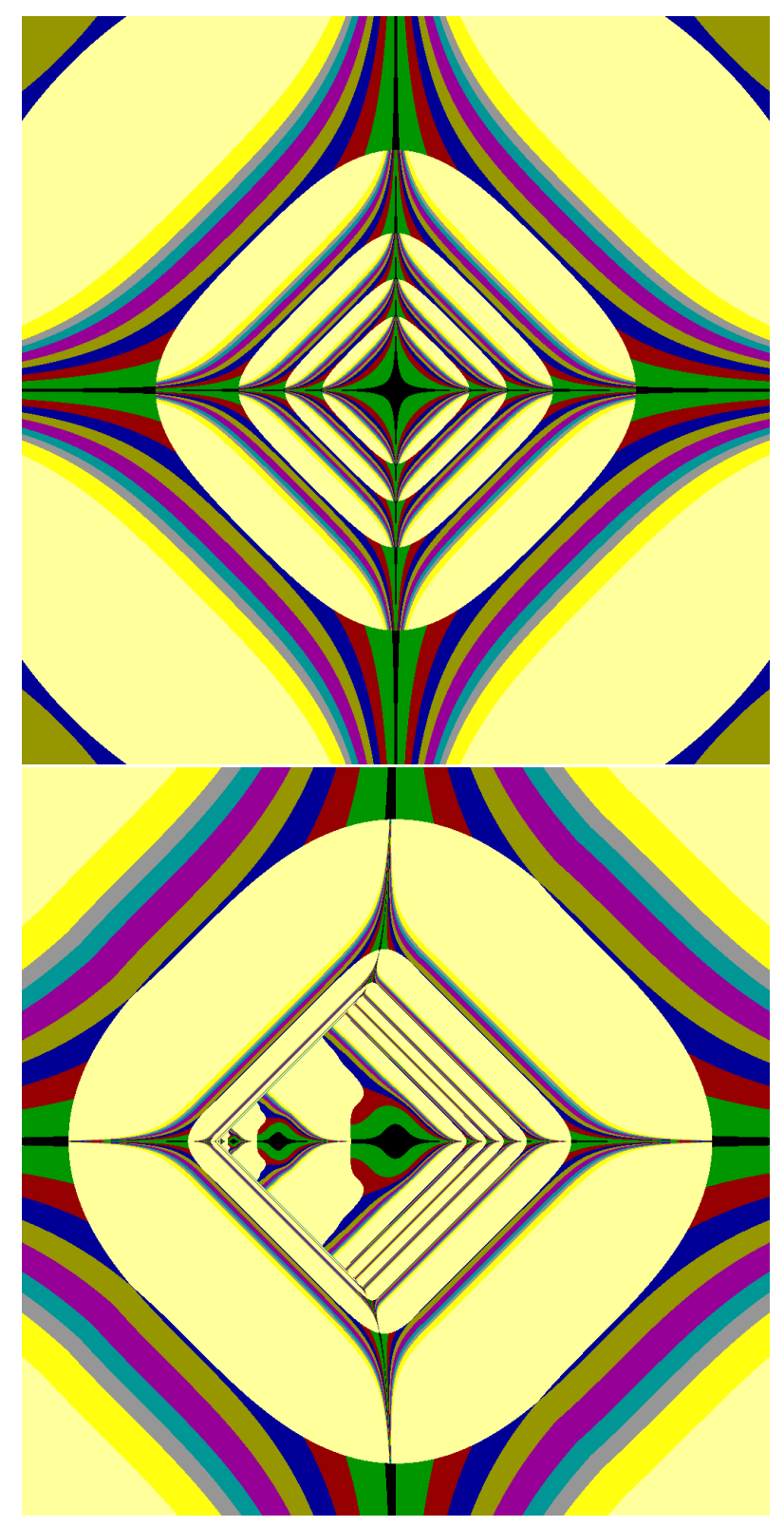

Figure 3: Pickover biomorphs for hyperbolic complex numbers.

The self-similarity though evident is clearly restricted and similar structures seem to be shifted and spatially separated from each other. 
3.4. Pickover Biomorphs for the Squares of Imaginary Units dependent on Points in the Complex Plane

The study has also experimented with a type of complex numbers with the following multiplication law:

$$
(a+b i)(c+d i)=\left(a c+b d i^{2}(a, b, c, d)\right)+(a d+b c) i,
$$

with the obvious requirement of symmetry $i^{2}(a, b, c, d)=i^{2}(c, d, a, b)$. The following images have been obtained from the iterations of a cubic function and three different Gaussian forms of $i^{2}$ depending only on $a$ and $c$. On the lefthand side of this figure $i^{2}=-1+\exp \left(-2\left(a^{2}+c^{2}\right)\right)$, on the right-hand side $i^{2}=\exp \left(-2\left(a^{2}+c^{2}\right)\right)$, and at the bottom $i^{2}=-1+2 \exp \left(-2\left(a^{2}+c^{2}\right)\right)$

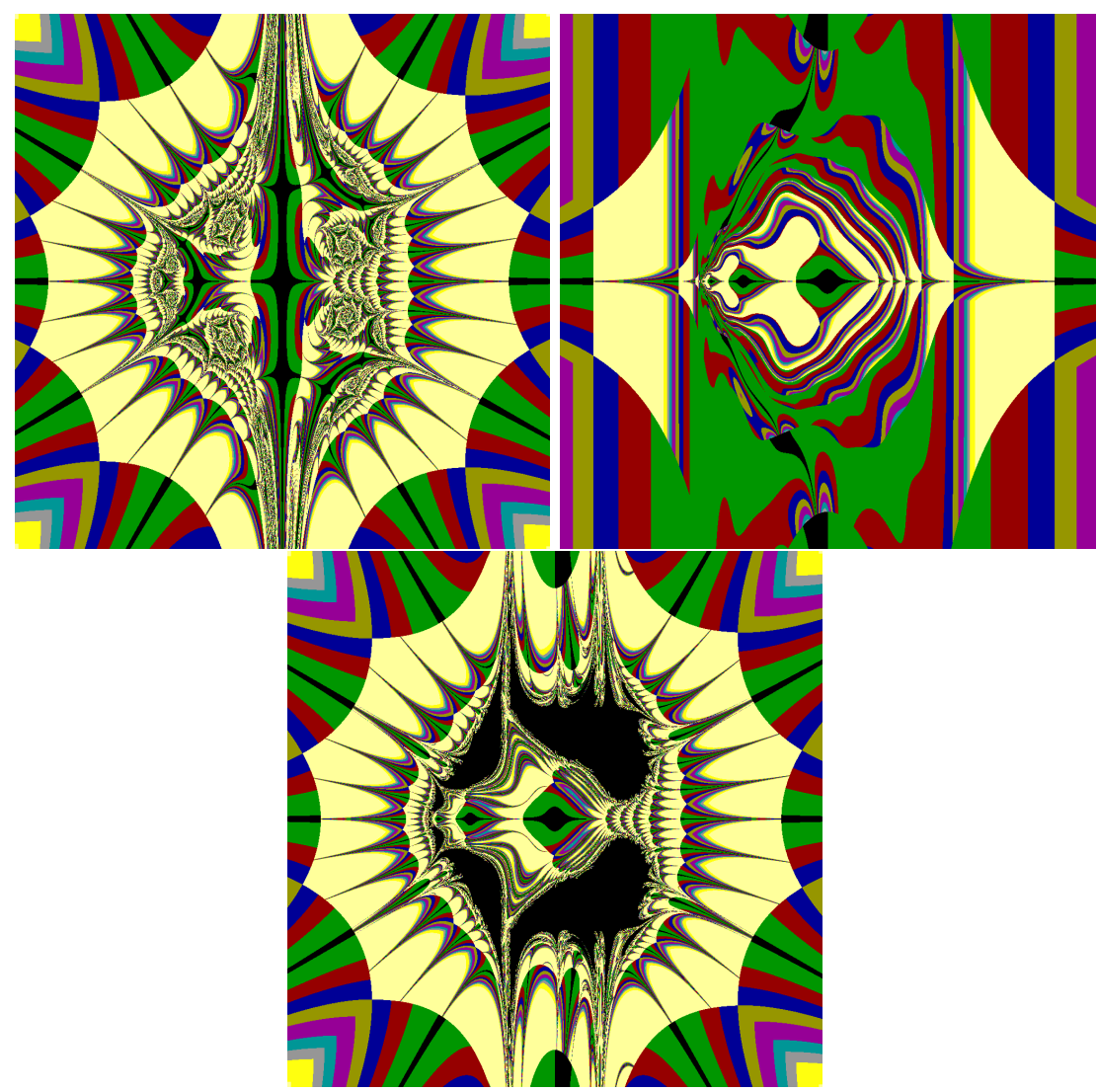

Figure 4: Pickover biomorphs obtained from the iteration of a cubic function for $i^{2}$ depending on points in the complex plane. 
Correlations in Images of Pickover Biomorphs

As a measure of correlations in the images of Pickover biomorphs Moran's autocorrelation function $I$ [19] has been chosen and is defined as:

$$
I=\frac{N}{W} \frac{\sum_{i, j} w_{i j}\left(x_{i}-\bar{x}\right)\left(x_{j}-\bar{x}\right)}{\sum_{i}\left(x_{i}-\bar{x}\right)^{2}},
$$

where $N$ is the number of spatial units indexed by $i$ and $j, \bar{x}$ is the mean of all $\left\{x_{i}\right\}, w_{i j}$ is a matrix of weights such that $w_{i i}=0$, and $W=\sum_{i, j} w_{i j}$. It is well known that the value of $I$ may strongly depend on the choice of matrix of weights. An attempt has not been made to obtain any comprehensive picture of such dependence in our case, but instead a simple binary matrix has been applied. Firstly, $w_{i j}$ was chosen to be 1 for the four nearest neighbours and 0 otherwise. Secondly, the computations were then repeated for $w_{i j}=1$ for the six nearest neighbours. These choices of neighbours correspond to the von Neumann and Moore neighbourhoods in cellular automata theory [20]. The biomorph for analysis was obtained using a grey-scale picture of the size of $1024 \times 1024$ pixels. 

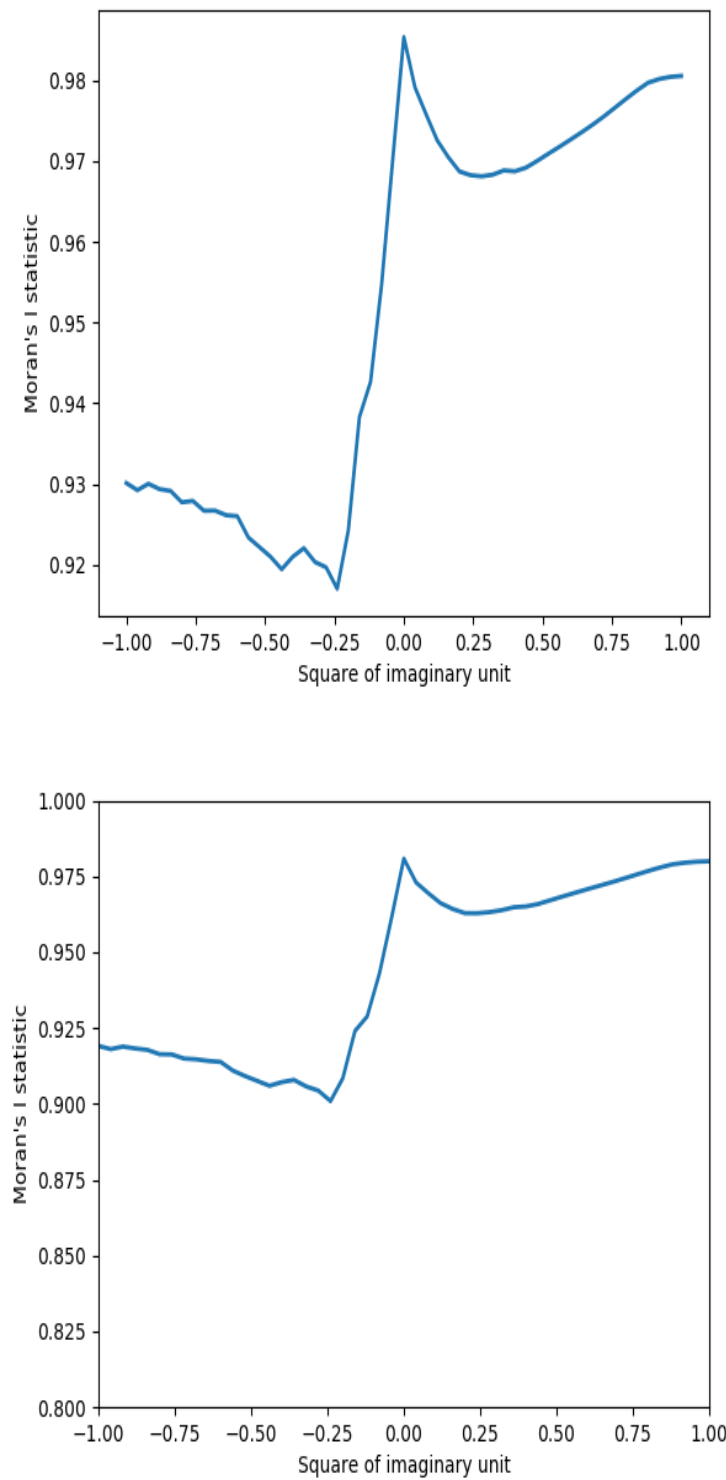

Figure 5: Moran's I global autocorrelation function as dependent on the square of the imaginary unit for a quadratic function. Top: Matrix of weights with 4 nearest neighbours; Bottom: Matrix of weights with 6 nearest neighbours. 

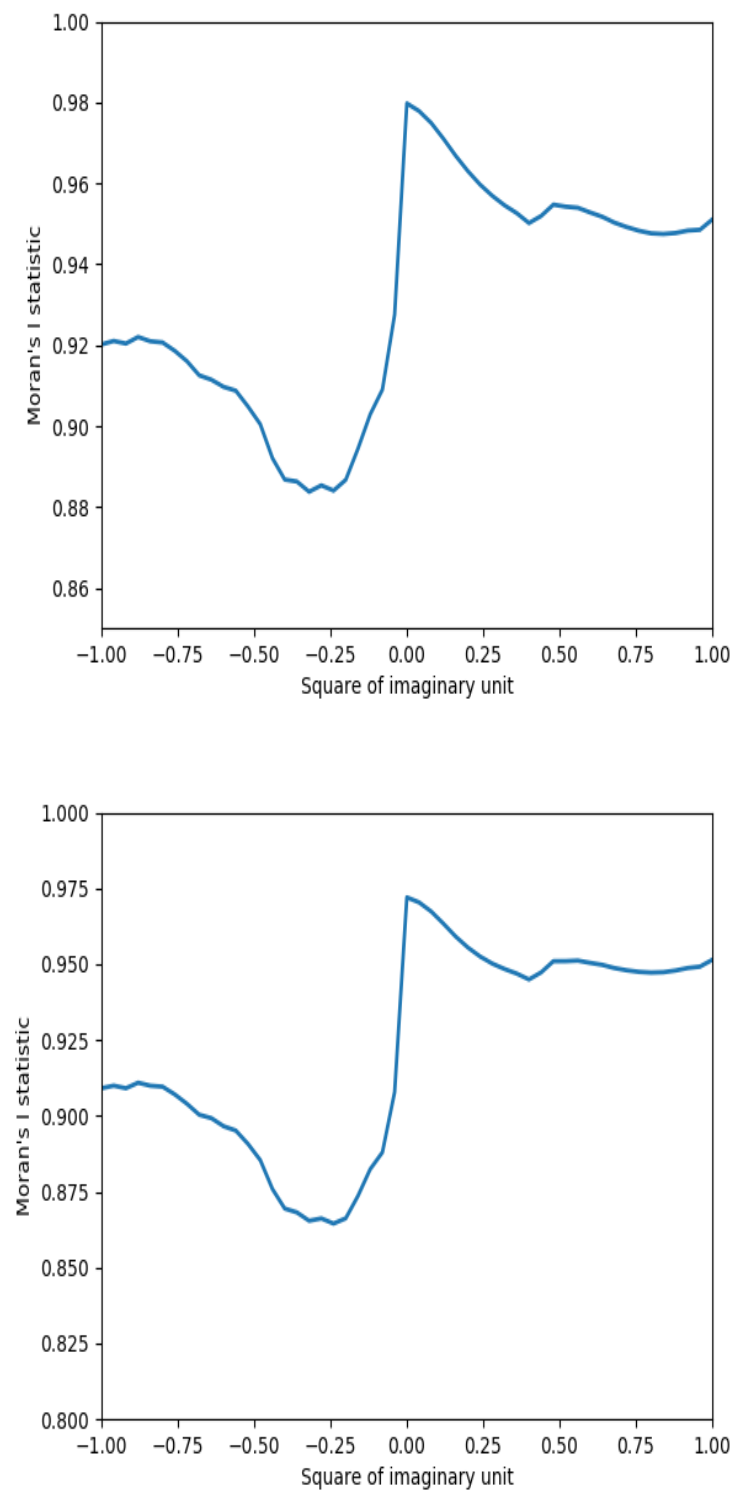

Figure 6: Moran's I global autocorrelation function as dependent on the square of the imaginary unit for a cubic function. Top: Matrix of weights with 4 nearest neighbours; Bottom: Matrix of weights with 6 nearest neighbours.

It appears that the correlations in biomorphs do not depend much on the system of weights in the Moran autocorrelation function. Furthermore, the qualitative outcome of the dependence on $i^{2}$ is the same for quadratic and cubic 
functions. We have observed in addition that Moran's function grows when the size of the pictures is increased from $128 x 128$ to $1024 x 1024$.

\subsection{Entropy as a Measure of Complexity in Images of Biomorphs}

To compute the global sample entropy [21] as well as the Shannon entropy of a biomorph, the matrix of grey-scale representation of a given image was used and then flattened to obtain a one-dimensional representation of the biomorph. This has been done for 50 values of the square of the imaginary unit in order to obtain a fairly comprehensive understanding both of the generic value of the entropy and its dependence on $i^{2}$. The results are shown in Figures 7 and 8 .
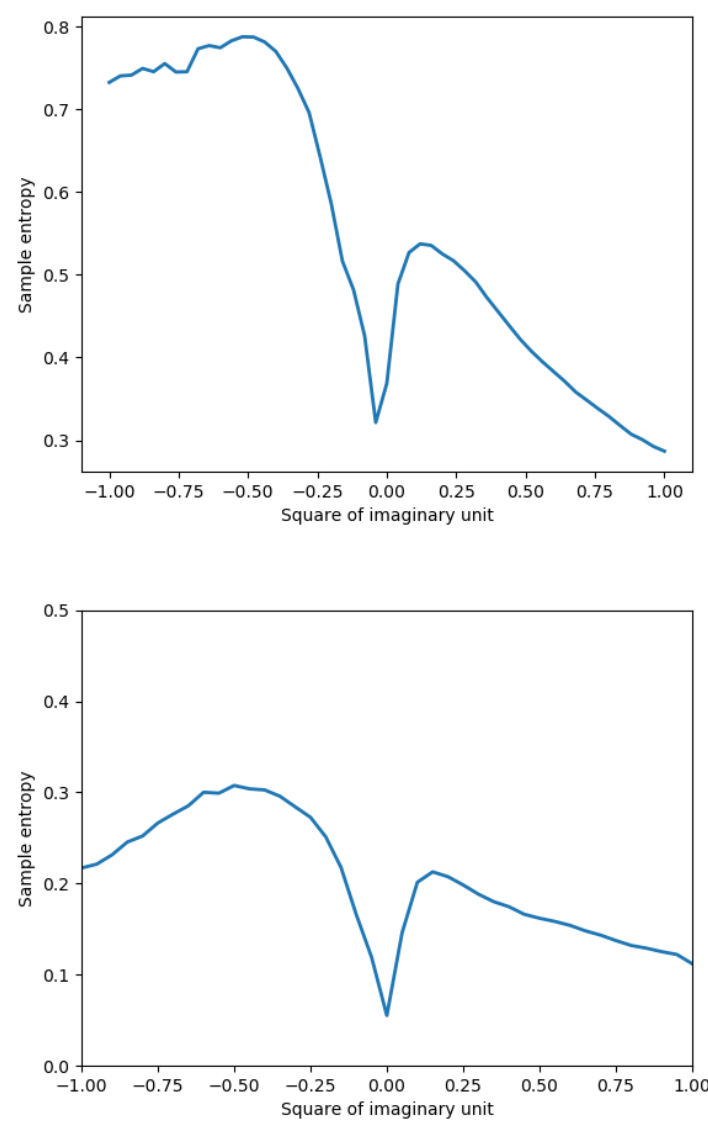

Figure 7: Sample Entropy as a Function of the Square of the Imaginary Unit. Top: for the quadratic function; Bottom: for the cubic function 


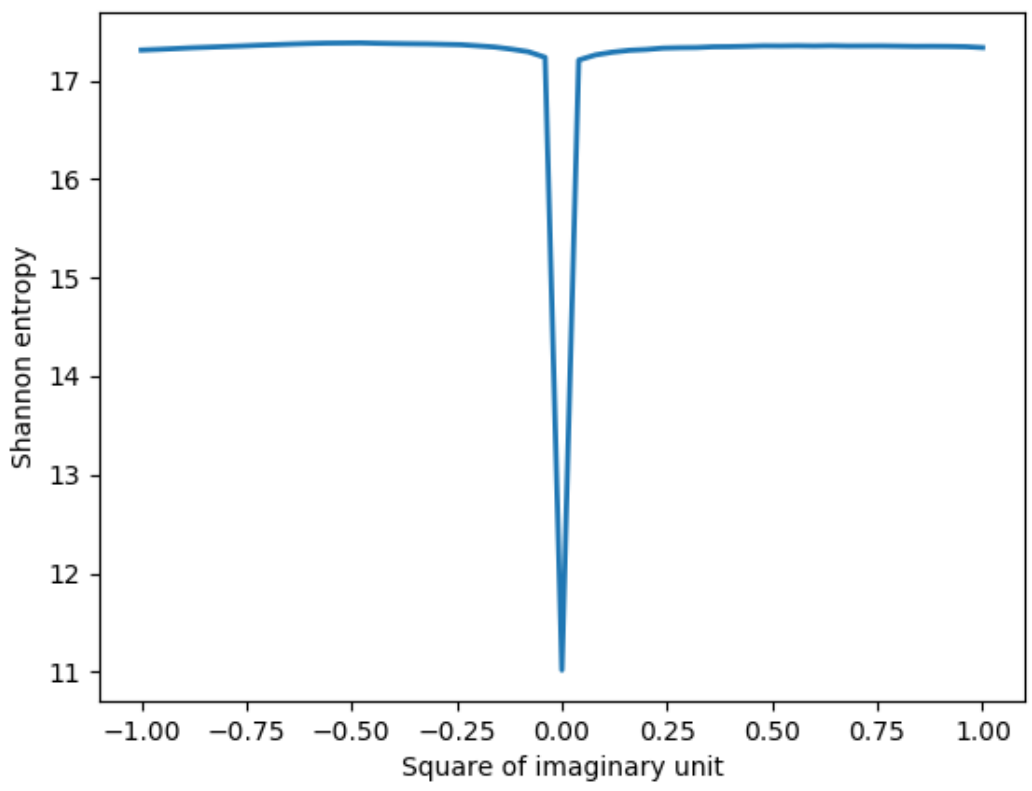

Figure 8: Shannon's entropy as a function of the square of the imaginary unit for the cubic function

In the calculations of sample entropy we have followed the standard prescriptions regarding the length $m$ of the data segment being compared (taken equal to 2) and similarity criterion $r$ (being equal to one fifth of the standard deviation). The sample entropy tends to be smaller for larger values of $i^{2}$, apart from the obvious local minimum near zero. Thus, the "time series" which represent the biomorph appear to have more repetitive patterns for those larger values. It is interesting to note that the Shannon entropy does not distinguish between the signs of $i^{2}$ and remains almost flat apart from the region close to $i^{2}=0$.

\subsection{Hurst Exponent}

The Hurst exponent is one of the measures of the long-term memory of a time series [22], [23]. It can be used as an indicator of irregularity, especially of time-series. Processes with higher (i.e. closer to 1) Hurst exponent appear to be more regular and indicate the presence of long-time correlations, [24]. Extension of the concept to any sequence of values (not necessarily labelled with time) is straightforward.

In our case, values of the Hurst exponent using the rescaled-range $R / S$ algorithms were also obtained through the use once again of a one-dimensional matrix obtained by flattening the original grey-scale representation of the biomorphs. The Hurst exponent has been computed from the resulting "time series". 

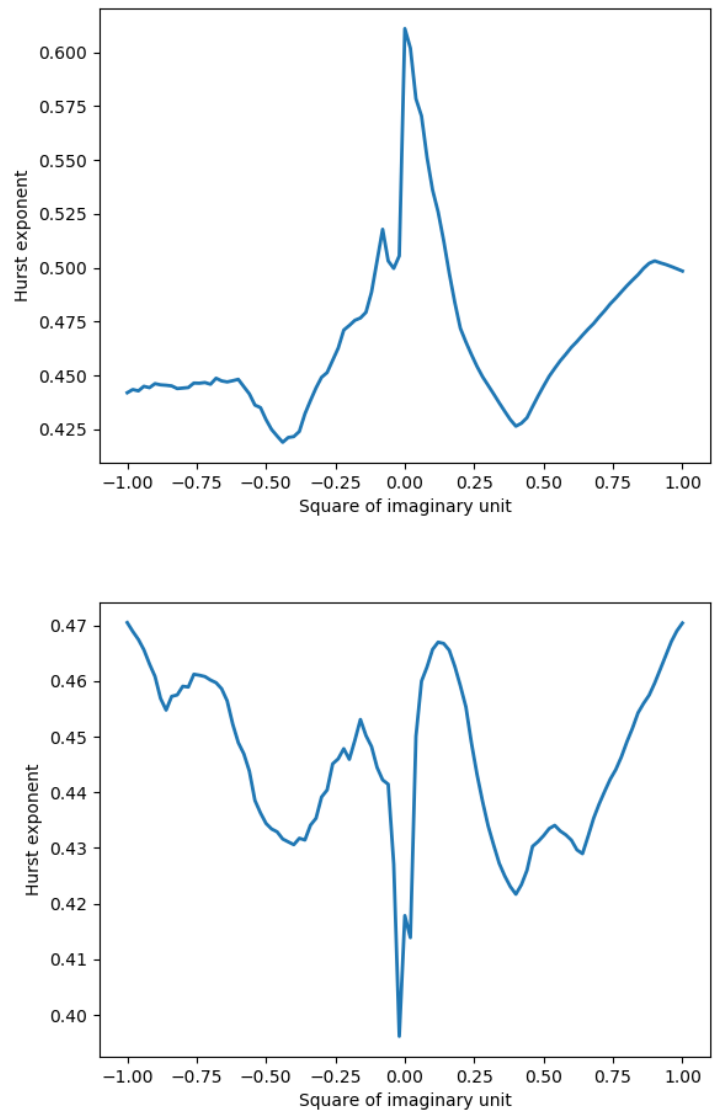

Figure 9: The Hurst exponent as a function of the square of the imaginary unit. A grey-scale representation of each biomorph was first obtained and then its matrix representation was flattened to obtain a one-dimensional sequence of values from which the Hurst exponent has been computed. Top: for the quadratic function; Bottom: for the cubic function.

A visible and interesting feature of the dependence of the Hurst exponent on $i^{2}$ is that it is very different for two iterated functions, and the structure of both dependences is rich with several minima and maxima. For the cubic function, for all values of $i^{2}$ the Hurst exponent has turned out to be less than 0.5 indicating that the fractal dimension of the one-dimensional representation of the biomorph may be between 1.5 and 2 with a strong maximum near $i^{2}=0$. But it is not true for the quadratic function, although the neighborhood of the point $i^{2}=0$ is clearly well distinguished.

It appears that the "time series" associated with the biomorphs are quite close to "Brownian motion" since the Hurst exponents are close to 0.5. However, weak "anti-persistency" is evident in the case of the cubic function. We have not yet found a proper interpretation of the details of the behaviour of Hurst 
exponents since more cases will need to be investigated to do this.

\section{Discussion}

In this work the properties of specific, biologically-inspired images obtained from iterations of complex functions have been analysed. These functions have been applied to three different systems of complex numbers: Euclidean (i.e. standard), Galilean, and hyperbolic complex numbers. The following conclusions can be drawn. Firstly, the concept of Pickover biomorphs can be extended from the set of complex numbers to the set of Galilean and hyperbolic complex numbers, and more generally to the set of numbers with the square of the imaginary unit being a function of points in the complex plane. Secondly, the graphical structures which emerge from iterations of (hyperbolic and Galilean) complex functions are usually simpler and less aesthetically appealing than in the case of standard (Euclidean) complex functions; however, self-similar structures are likely to appear. Thirdly, several measures of correlations as well as complexity such as Moran's correlation function, sample entropy and the Hurst exponent can be useful in the analysis of biomorphs associated with any concept of complex numbers. Fourthly, whilst all the biomorphs are graphical objects in which naturally, strong spatial correlations appear, the latter are less pronounced in the case of standard (Euclidean) complex numbers than in the case of hyperbolic numbers with a somewhat unexpected deep local minimum on the side of the negative values of the square of the imaginary unit $i^{2}$, and a strong maximum very close to the zero value of $i^{2}$. Fifthly, the dependence of sample entropy (obtained by flattening the matrix representation of the grey-scale image representing a biomorph) appears to be complementary with respect to the Moran autocorrelation function. The sample entropy is smaller for Galilean and hyperbolic numbers than for Euclidean ones, indicating stronger self-similarity in the former case. This is, however, mostly due to the higher overall symmetry of biomorphs obtained with non-negative $i^{2}$. Finally, the Hurst exponent has been obtained as a function of $i^{2}$.

\section{Methods}

All programs for the purpose of this work have been written in Python (version 2.7). The following Python modules have been used: numpy, scipy, matplotlib, Pillow [25], pysal [26], pyentrp [27], and nolds [28].

In explanation of the process of the generation of the figures, the drawing area of $1024 \times 1024$ pixels was chosen in order to obtain the drawings of the biomorphs. The parameter $C$ which enters the iteration algorithm was set equal to $(0.5,0)$ and the drawing area was mapped to an area of (generalized) complex plane of the size $([-2.5,2.5] x[-2.5,2.5])$. The maximal number of iterations has been set equal to 100; the programs left the iteration loops if the iteration index exceeded 100, or if either the real or the imaginary part of $z$ exceeded 10.0. Then the final values of the real and the imaginary parts of $z$ were tested for 
whether any of them belong to an interval $(a, b)$. Depending on $(a, b)$, a specific colour of the pixel corresponding to the initial value of $z$ has been chosen as illustrated by the following tables:

Table 1: Colouring code for the biomorphs obtained on using standard (Euclidean) complex numbers (1)

\begin{tabular}{|c|c|c|}
\hline $\mathrm{a}$ & $\mathrm{b}$ & $\mathrm{RGB}$ \\
\hline 0.0 & 0.5 & $(0,0,0)$ \\
0.5 & 4.0 & $(0,150,0)$ \\
4.0 & 7 & $(150,0,0)$ \\
7.0 & 10.0 & $(0,0,150)$ \\
10.0 & 13.0 & $(150,150,0)$ \\
13.0 & 17.0 & $(150,0,150)$ \\
17.0 & 20.0 & $(0,150,150)$ \\
20.0 & 23.0 & $(150,150,150)$ \\
23.0 & $\infty$ & $(255,255,155)$ \\
\hline
\end{tabular}

Table 2: Colouring code for the biomorphs obtained on using Galilean complex numbers (2)

\begin{tabular}{|c|c|c|}
\hline $\mathrm{a}$ & $\mathrm{b}$ & $\mathrm{RGB}$ \\
\hline 0.0 & $10^{-10}$ & $(50,50,50)$ \\
$10^{-10}$ & 1.0 & $(0,150,0)$ \\
1.0 & 2.5 & $(150,0,0)$ \\
2.5 & 4.0 & $(0,0,150)$ \\
4.0 & 5.0 & $(150,150,0)$ \\
5.0 & 6.0 & $(150,0,150)$ \\
6.0 & 7.0 & $(0,150,150)$ \\
7.0 & 8.0 & $(255,0,15)$ \\
8.0 & $\infty$ & $(255,255,155)$ \\
\hline
\end{tabular}

Table 3: Colouring code for the biomorphs obtained on using hyperbolic complex numbers (3)

\begin{tabular}{|c|c|c|}
\hline $\mathrm{a}$ & $\mathrm{b}$ & $\mathrm{RGB}$ \\
\hline 0.0 & 0.5 & $(0,0,0)$ \\
0.5 & 4.0 & $(0,150,0)$ \\
4.0 & 7.0 & $(150,0,0)$ \\
7.0 & 10.0 & $(0,0,150)$ \\
10.0 & 13.0 & $(150,150,0)$ \\
13.0 & 17.0 & $(150,0,150)$ \\
17.0 & 20.0 & $(0,150,150)$ \\
20.0 & 23.0 & $(150,150,150)$ \\
23.0 & 30.0 & $(250,250,15)$ \\
30.0 & $\infty$ & $(255,255,155)$ \\
\hline
\end{tabular}


In the above tables, "RGB" signifies the amount of the Red, Green, and Blue colours in the colour of a pixel. The code of 3 has also been used to produce 4 .

[1] B. B. Mandelbrot, The Fractal Geometry of Nature, W.H. Freeman, New York, 1983.

[2] K. J. Falconer, Fractal geometry; mathematical foundations and applications, Wiley, Chichester, 1990.

[3] M. F. Barnsley, Fractals everywhere, Academic Press, New York, 1988.

[4] J. Kudrewicz, Fractals and Chaos [in Polish], WNT, Warsaw, 1993.

[5] C. A. Pickover, Computers, Pattern, Chaos and Beauty, Dover, New York, 2001.

[6] C. A. Pickover, Chaos, complexity and accident, YLEM Newsletter 19(12) (1999) 1-6.

[7] C. A. Pickover, The Pattern Book: Fractals, Art and Nature, World Scientific, Singapore, 1995.

[8] C. A. Pickover, Chaos and Fractals: A Computer Graphical Journey, Elsevier, Amsterdam, 1998.

[9] K. Gdawiec, W. Kotarski, A. Lisowska, Biomorphs via modified iterations, Journal of Nonlinear Science and Applications 9(5) (2016) 2305-2315.

[10] N. S. Mojica, J. Navarro, P. C. Marijuan, R. Lahoz-Beltra, Cellular "bauplans": evolving unicellular forms by means of Julia sets and Pickover biomorphs, Biosystems 98 (2009) 19-30.

[11] M. Levin, Morphogenetic fields in embryogenesis, regeneration, and cancer: non-local control of complex patterning, Biosystems 109 (2012) 243-261.

[12] A. F. Beardon, Iteration of Rational Functions, Springer, Berlin, 1991.

[13] J. Hucks, Hyperbolic complex structures in physics, Journal of Mathematical Physics 34 (1993) 5986.

[14] I. M. Yaglom, Complex Numbers and Their Application in Geometry [in Russian], LIBROKOM, Moscow, 2009.

[15] C. K. Law, Resonance response of the quantum vacuum to an oscillating boundary, Phys. Rev. Lett. 73 (1994) 1931-1934.

[16] M. Janowicz, Evolution of wave fields and atom-field interactions in a cavity with one oscillating mirror, Phys. Rev. A 57 (1998) 4784-4790. 
[17] V. V. Dodonov, A. A. Andreata, Pulse generation in vibrating cavities, In: Coherence and Quantum Optics VIII, Proceedings of the Eighth Rochester Conference on Coherence and Quantum Optics, eds. N.P. Bigelow, J.H. Eberly, C.R. Stroud Jr, L.A. Walmsley (Kluwer Academic/Plenum Publishers) VIII (2003) 497-498.

[18] D. E. Knuth, The Art of Computer Programming, Addison-Wesley, New York, 1997.

[19] P. A. P. Moran, Notes on continuous stochastic phenomena, Biometrika 37 (1950) 17-23.

[20] A. Ilachinski, Cellular Automata. A Discrete Universe, World Scientific, Singapore, 2001.

[21] J. S. Richman, J. R. Moorman, Physiological time-series analysis using approximate entropy and sample entropy, American Journal of Physiology. Heart and Circulatory Physiology 278(6) (2000) H203949.

[22] H. E. Hurst, Long-term storage capacity of reservoirs, Transactions of American Society of Civil Engineers 116 (1951) 770.

[23] B. B. Mandelbrot, J. R. Wallis, Noah, joseph, and operational hydrology, Water Resour. Res. 4 (1968) 909-918.

[24] E. E. Peters, Fractal Market Analysis: Applying Chaos Theory to Investment and Economics., Wiley, New York, 1994.

[25] F. Lundh, C. A., Pillow (2017).

URL https://pillow.readthedocs.io/en/4.3.x/

[26] S. J. Rey, L. Anselin, Pysal (2017).

URL http://pysal.readthedocs.io/en/latest/index.html

[27] Donets, Pyentrp (2017).

URL https://pypi.python.org/pypi/pyentrp/0.3.1

[28] C. Schölzl, Nolds - nonlinear measures for dynamical systems (based on one-dimensional time series) (2017).

URL https://pypi.python.org/pypi/nolds/0.4.0

\section{Acknowledgements}

The authors would like to thank Dr Magorzata Dudkiewicz for interesting discussions on the paper. This work was supported by the University of Wrocaw under Grant No. 0401/0449/18. 